\title{
Direct Fisher Inference of the Quartic Oscillator's Eigenvalues
}

\author{
S. P. Flego ${ }^{1}$, Angelo Plastino ${ }^{2,5}$, A. R. Plastino ${ }^{3,4}$ \\ ${ }^{1}$ Universidad Nacional de La Plata, Facultad de Ingeniera, Área Departamental de \\ Ciencias Básicas, La Plata, Argentina \\ ${ }^{2}$ Universidad Nacional de La Plata, Instituto de Fsica (IFLP-CCT-CONICET), La Plata, Argentina \\ ${ }^{3}$ CREG-Universidad Nacional de La Plata-CONICET, La Plata, Argentina \\ ${ }^{4}$ Instituto Carlos I de Fisica Teorica y Computacional and Departamento de Fisica Atomica, \\ Molecular y Nuclear, Universidad de Granada, Granada, Spain \\ ${ }^{5}$ Universitat de les Illes Balears and IFISC-CSIC, Palma de Mallorca, Spain \\ E-mail: angeloplastino@gmail.com \\ Received June 22, 2011; revised July 30, 2011; accepted August 23, 2011
}

\begin{abstract}
It is well known that a suggestive connection links Schrödinger's equation (SE) and the information-optimizing principle based on Fisher's information measure (FIM). It has been shown that this entails the existence of a Legendre transform structure underlying the SE. Such a structure leads to a first order partial differential equation (PDE) for the SE's eigenvalues from which a complete solution for them can be obtained. We test this theory with regards to anharmonic oscillators (AHO). AHO pose a long-standing problem and received intense attention motivated by problems in quantum field theory and molecular physics. By appeal to the Cramer Rao bound we are able to Fisher-infer the energy eigenvalues without explicitly solving Schrödinger's equation. Remarkably enough, and in contrast with standard variational approaches, our present procedure does not involve free fitting parameters.
\end{abstract}

Keywords: Information Theory, Fisher's Information Measure, Legendre Transform, Quartic Anharmonic Oscillator

\section{Introduction}

It is well-known that a strong link exists between Fisher’ information measure (FIM) $I[1]$ and Schrödinger wave equation (SE) [2-7]. In a nutshell, this connection is based upon the fact that a constrained Fisher-minimizetion leads to a SE-like equation [1-7]. In turn, this implies the existence of intriguing relationships between various SE-facets, and Jaynes's maximum entropy principle. In particular, basic SE-consequences such as the Hellmann-Feynman and the Virial theorems can be reinterpreted in terms of a special kind of reciprocity relations between relevant physical quantities, similar to the ones exhibited by the thermodynamics' formalism via its Legendre-invariance property $[5,6]$. This fact demonstrates that a Legendre-transform structure underlies the non-relativistic Schrödinger equation. As a consequence, the possible energy-eigenvalues are now seen to be constrained by such structure in a rather unsuspected way
[5-7], a fact that allows one to obtain a first-order differential equation, unrelated to Schroedinger's equation [7], that energy eigenvalues must necessarily satisfy. The predictive power of this new equation will be explored here.

We will apply our formalism here to the quantum anharmonic oscillator, which is the paradigmatic testingground for new approaches to Schroedinger eigenvalue equation. Besides their intrinsic conceptual and mathematical interest, anharmonic oscillators have received considerable attention over the years due to their practical relevance in connection with several areas of physics, such as quantum field theory and molecular physics, among others. In this kind of systems, the most intense focus has been traditionally concentrated upon the quartic oscillator. General accounts containing illuminating references on this problem may be found, for instance, in [8]. Note that a perturbation series solution to this problem in powers of the anharmonicity-parameter $\lambda$ is 
divergent-asymptotic for all $\lambda>0$ [9]. Specifically, we will apply our procedure to treat the quartic anharmonic oscillator. By appeal to the Cramer Rao bound we obtain the particular solution that leads to the system's eigenvalues without need of explicitly solving Schrödinger's equation. More importantly, we do not need to appeal to any arbitrary or empirical parameter, as is common practice in other treatments [10]. The parameter-free nature of our treatment is evidence of the structural physical information that we incorporate to the theory via Fisher's information measure.

\section{Basic Ideas}

Let $x$ be a stochastic variable and $f(x, \theta)$ the probability density function (PDF) for this variable, which depends on the parameter $\theta$. If an observer were to make a measurement of $x$ and had to best infer $\theta$ from such measurement, calling the resulting estimate $\tilde{\theta}=\tilde{\theta}(x)$, one might ask how well could $\theta$ be determined. Estimation theory [1] tells us that the best possible estimator $\tilde{\theta}(x)$, after a very large number of $x$-samples is examined, suffers a mean-square error $\Delta x$ from $\theta$ obeying the rule $I(\Delta x)^{2}=1$, where $I$ is an information quantifier called the Fisher information measure (FIM), a non linear functional of the PDF that reads

$$
I=\int \mathrm{d} x f(x, \theta)\left\{\frac{\partial}{\partial \theta} \ln [f(x, \theta)]\right\}^{2} .
$$

Any other estimator must have a larger mean-square error (all estimators must be unbiased, i.e., satisfy $\langle\tilde{\theta}(x)\rangle=\theta$ ). Thus, FIM has a lower bound. No matter what the parameter $\theta$ might be, $I$ has to obey

$$
I(\Delta x)^{2} \geq 1,
$$

the celebrated Cramer-Rao bound [1].

In the case of physical Fisher applications, the particular instance of translational families has received a great deal of attention in the literature [1]. These are mono-parametric distribution families of the form $f(x, \theta)=f(x-\theta)$, known up to the shift parameter $\theta$. All family members exhibit identical shape. For such families one gets

$$
I=\int f(x)\left(\frac{\partial \ln f(x)}{\partial x}\right)^{2} \mathrm{~d} x
$$

Focus attention now a system that is specified by a set of $M$ physical parameters $\mu_{k}$. We can write $\mu_{k}=\left\langle A_{k}\right\rangle$, with $A_{k}=A_{k}(x)$. The set of $\mu_{k}$-values is to be regarded as our prior knowledge. It represents our available empirical information. Let the pertinent probability distribution function (PDF) be $f(x)$. Then,

$$
\left\langle A_{k}\right\rangle=\int \mathrm{d} x A_{k}(x) f(x), k=1, \cdots, M .
$$

In this context it can be shown (see for example [2,3]) that the physically relevant PDF $f(x)$ minimizes FIM subject to the prior conditions and the normalization condition. Normalization entails $\int \mathrm{d} x f(x)=1$, and, consequently, our Fisher-based extremization problem adopts the appearance

$$
\delta\left(I-\alpha \int \mathrm{d} x f(x)-\sum_{k=1}^{M} \lambda_{k} \int \mathrm{d} x A_{k}(x) f(x)\right)=0,
$$

where we have introduced the $(M+1)$ Lagrange multipliers $\lambda_{k}\left(\lambda_{0}=\alpha\right)$. In Ref. [2] on can find the details of how to go from (5) to a Schrödinger's equation (SE) that yields the desired PDF in terms of the amplitude $\psi(x)$ defined by $f(x)=\psi(x)^{2}$. This SE is of the form

$$
\begin{aligned}
& {\left[-\frac{1}{2} \frac{\partial^{2}}{\partial x^{2}}+U(x)\right] \psi=\frac{\alpha}{8} \psi,} \\
& U(x)=-\frac{1}{8} \sum_{k=1}^{M} \lambda_{k} A_{k}(x),
\end{aligned}
$$

and can be formally interpreted as the (real) Schrödinger equation (SE) for a particle of unit mass ( $\hbar=1$ ) moving in the effective, "information-related pseudo-potential" $U(x)$ [2] in which the normalization-Lagrange multiplier $(\alpha / 8)$ plays the role of an energy eigenvalue. The $\lambda_{k}$ are fixed, of course, by recourse to the available prior information. In the case of one-dimensional scenarios, $\psi(x)$ is real [11] and

$$
\begin{aligned}
I & =\int \psi^{2}\left(\frac{\partial \ln \psi^{2}}{\partial x}\right)^{2} \mathrm{~d} x=4 \int\left(\frac{\partial \psi}{\partial x}\right)^{2} \mathrm{~d} x \\
& =-4 \int \psi \frac{\partial^{2}}{\partial x^{2}} \psi \mathrm{d} x
\end{aligned}
$$

so that using the SE (6) we obtain

$$
I=\alpha+\sum_{k=1}^{M} \lambda_{k}\left\langle A_{k}\right\rangle
$$

\section{Legendre Structure}

The connection between the variational solutions $f$ and thermodynamics was established in Refs. [2] and [4] in the guise of reciprocity relations that express the Legendre-transform structure of thermodynamics. They constitute its essential formal ingredient [12] and were rederived à la Fisher in [2] by recasting (8) in a fashion that emphasizes the role of the relevant independent variables,

$$
I\left(\left\langle A_{1}\right\rangle, \cdots,\left\langle A_{M}\right\rangle\right)=\alpha+\sum_{k=1}^{M} \lambda_{k}\left\langle A_{k}\right\rangle .
$$

Obviously, the Legendre transform main goal is that of 
changing the identity of our relevant variables. As for $I$ we have

$$
\alpha\left(\lambda_{1}, \cdots, \lambda_{M}\right)=I-\sum_{k=1}^{M} \lambda_{k}\left\langle A_{k}\right\rangle,
$$

so that we encounter the three reciprocity relations (proved in [2])

$$
\frac{\partial \alpha}{\partial \lambda_{k}}=-\left\langle A_{k}\right\rangle ; \frac{\partial I}{\partial\left\langle A_{k}\right\rangle}=\lambda_{k} ; \frac{\partial I}{\partial \lambda_{i}}=\sum_{k}^{M} \lambda_{k} \frac{\partial\left\langle A_{k}\right\rangle}{\partial \lambda_{i}},
$$

the last one being a generalized Fisher-Euler theorem.

\section{Fisher Measure and Quantum Mechanical Connection}

Since $U(x)$ belongs to $\mathcal{L}_{2}$ and thus admits of a series expansion in the powers-of- $x$-basis $x, x^{2} x^{3}$, etc. [13]. Of course, the $A_{k}(x)$ themselves also belong to $\mathcal{L}_{2}$. They can thus be series-expanded in similar fashion, which enables one to base future considerations on the hypothesis that the prior-knowledge refers to powers $x^{k}$, i.e., $\left\langle A_{k}\right\rangle=\left\langle x^{k}\right\rangle$. Assume then that one has gathered information concerning $M$ moments $\left\langle x^{k}\right\rangle$. The "information” potential $U$ acquires the aspect

$$
U(x)=-\frac{1}{8} \sum_{k} \lambda_{k} x^{k}
$$

and we consider that the first $M$ summands suffice to obtain a satisfactory representation of $U(x)$. Of course, our Lagrange multipliers are now to be identified with $U(x)$ 's series-expansion's coefficients.

For such Schrödinger-platform the virial theorem reads [5]

$$
\left\langle\frac{\partial^{2}}{\partial x^{2}}\right\rangle=-\left\langle x \frac{\partial}{\partial x} U(x)\right\rangle=\frac{1}{8} \sum_{k=1}^{M} k \lambda_{k}\left\langle x^{k}\right\rangle,
$$

and thus, from (7) and (13) a virial-related expression for Fisher's information measure can be obtained [5] in the fashion

$$
I=-\sum_{k=1}^{M} \frac{k}{2} \lambda_{k}\left\langle x^{k}\right\rangle,
$$

Now, substituting such $I$-form into (8) and solving for $\alpha$ we find

$$
\alpha=-\sum_{k=1}^{M}\left(1+\frac{k}{2}\right) \lambda_{k}\left\langle x^{k}\right\rangle .
$$

$\alpha(I)$ is explicit function of the $M$ Lagrange multipliers $-U(x)$ 's series-expansion coefficients $\lambda_{k}$ (the physical parameters $\left\langle x^{k}\right\rangle$ ). Equations (14) and (15) encode in adequate fashion the information provided by the virial theorem $[5,6]$.

\subsection{Fisher-Schröedinger Legendre Structure}

Interestingly enough, the reciprocity relations (RR) (11) can be re-derived on a strictly pure quantum mechanical basis [5], starting from

1) the quantum Virial theorem [which leads to Equations (14) and (15)] plus

2) information provided by the quantum HellmannFeynman theorem.

This fact indicates that a Legendre structure underlays the one-dimensional Schröedinger equation [5]. Thus, with $\left\langle A_{k}\right\rangle=\left\langle x^{k}\right\rangle$, our "new" reciprocity relations are given by

$$
\frac{\partial \alpha}{\partial \lambda_{k}}=-\left\langle x^{k}\right\rangle ; \frac{\partial I}{\partial\left\langle x^{k}\right\rangle}=\lambda_{k} ; \frac{\partial I}{\partial \lambda_{i}}=\sum_{k}^{M} \lambda_{k} \frac{\partial\left\langle x^{k}\right\rangle}{\partial \lambda_{i}},
$$

FIM expresses a relation between the independent variables or control variables (the prior information) and $I$. Such information is encoded into the functional form $I=I\left(\left\langle x^{1}\right\rangle, \cdots,\left\langle x^{M}\right\rangle\right)$. For later convenience, we will also denote such a relation or encoding as $\left\{I,\left\langle x^{k}\right\rangle\right\}$. We see that the Legendre transform FIM-structure involves eigenvalues of the "information-Hamiltonian" and Lagrange multipliers. Information is encoded in $I$ via these Lagrange multipliers, i.e., $\alpha=\alpha\left(\lambda_{1}, \cdots, \lambda_{M}\right)$, together with a bigection $\left\{I,\left\langle x^{k}\right\rangle\right\} \leftrightarrow\left\{\alpha, \lambda_{k}\right\}$.

\subsection{Two Scenarios}

In a $\left\{\boldsymbol{I},\left\langle\boldsymbol{x}^{\boldsymbol{k}}\right\rangle\right\}$-scenario, the $\lambda_{k}$ are functions dependent on the $\left\langle x^{k}\right\rangle$-values. As shown in [6], substituting the RR given by (16) in (14) one is led to a linear, partial differential equations (PDE) for $I$,

$$
\lambda_{k}=\frac{\partial I}{\partial\left\langle x^{k}\right\rangle} \rightarrow I=-\sum_{k=1}^{M} \frac{k}{2}\left\langle x^{k}\right\rangle \frac{\partial I}{\partial\left\langle x^{k}\right\rangle} .
$$

and a complete solution is given by

$$
I\left(\left\langle x^{1}\right\rangle, \cdots,\left\langle x^{M}\right\rangle\right)=\sum_{k=1}^{M} C_{k}\left|\left\langle x^{k}\right\rangle\right|^{-2 / k},
$$

where $C_{k}$ are positive real numbers (integration constants). The $I$ - domain is

$D_{I}=\left\{\left(\left\langle x^{1}\right\rangle, \cdots,\left\langle x^{M}\right\rangle\right) /\left\langle x^{k}\right\rangle \in \mathfrak{R}_{o}\right\}$. Equation (18) states that for $\left\langle x^{k}\right\rangle>0, I$ is a monotonically decreasing function of $\left\langle x^{k}\right\rangle$, and as one expects from a "good" information measure [1], $I$ is a convex function. We may obtain $\lambda_{k}$ from the reciprocity relations (16). For $\left\langle x^{k}\right\rangle>0$ one gets, 


$$
\lambda_{k}=\frac{\partial I}{\partial\left\langle x^{k}\right\rangle}=-\frac{2}{k} C_{k}\left\langle x^{k}\right\rangle^{-(2+k) / k}<0 .
$$

and then, using (8), we obtain the $\alpha$-normalization Lagrange multiplier.

The general solution for the I-PDE does exist and its uniqueness has been demonstrated via an analysis of the associated Cauchy problem [6]. Thus, Equation (18) implies what seems to be a kind of "universal" prescription, a linear PDE that any variationally (with constraints) obtained FIM must necessarily comply with.

In the $\left\{\boldsymbol{\alpha}, \lambda_{k}\right\}$ scenario, the $\left\langle x^{k}\right\rangle$ are functions that depend on the $\lambda_{k}$-values. As we showed in [7], an analog $\alpha$-PDE exists. Substituting the RR given by (16) in (15) we are led to

$$
\frac{\partial \alpha}{\partial \lambda_{k}}=-\left\langle x^{k}\right\rangle \rightarrow \alpha=\sum_{k=1}^{M}\left(1+\frac{k}{2}\right) \lambda_{k} \frac{\partial \alpha}{\partial \lambda_{k}} .
$$

and a complete solution is given by

$$
\alpha\left(\lambda_{1}, \cdots, \lambda_{M}\right)=\sum_{k=1}^{M} D_{k}\left|\lambda_{k}\right|^{2 /(2+k)},
$$

where the $D_{k} \mathrm{~s}$ are positive real numbers (integration constants). The $\alpha$-domain is

$D_{\alpha}=\left\{\left(\lambda_{1}, \cdots, \lambda_{M}\right) / \lambda_{k} \in \mathfrak{R}\right\}=\mathfrak{R}^{M}$. Also, Equation (21) states that for $\lambda_{k}<0, \alpha$ is a monotonically decreasing function of the $\lambda_{k}$, and as one expect from the Legendre transform of $I$, we end up with a concave function. We may obtain the $\left\langle x^{k}\right\rangle$ 's from the reciprocity relations (16). For $\lambda_{k}<0$ one gets

$$
\left\langle x^{k}\right\rangle=-\frac{\partial \alpha}{\partial \lambda_{k}}=\frac{2}{(2+k)} D_{k}\left|\lambda_{k}\right|^{-k /(2+k)}>0 .
$$

and then, using (8) one us able to build up $I$.

The general solution for $\alpha$ - PDE exists. Uniqueness is, again, proved from an analysis of the associated Cauchy problem [7]. Thus, Equation (18) implies once more a kind of "universal" prescription, a linear PDE that all SE-eigenvalues must necessarily comply with.

The mathematical structure of the Legendre transform leads to a relation between the integration constants $C_{k}$ and $D_{k}$ pertaining to the $I$ and $\alpha$ expressions, respectively, given by (18) and (21). In [7] we studied with some detail this relation. In our two scenarios, $\left\{I,\left\langle x^{k}\right\rangle\right\}$ and $\left\{\alpha, \lambda_{k}\right\}$, we have [7]

$$
C_{k}=\frac{k}{2} \bar{C}_{k}, D_{k}=\frac{k+2}{2} \bar{D}_{k} \text {, with } \bar{D}_{k}^{(2+k)}=\bar{C}_{k}^{k} \equiv F_{k}^{2} \text {. }
$$

Consequently, expressions (18) and (21) take the form,

$$
I=\sum_{k=1}^{M} \frac{k}{2}\left[\frac{F_{k}}{\mid\left\langle x^{k}\right\rangle}\right]^{2 / k}, \alpha=\sum_{k=1}^{M} \frac{k+2}{2}\left[F_{k}\left|\lambda_{k}\right|\right]^{2 /(2+k)} \text {. }
$$

The reciprocity relations (19) and (22) can thus be economically summarized in the fashion

$$
F_{k}^{2}=\left|\lambda_{k}\right|^{k}\left|\left\langle x^{k}\right\rangle\right|^{(2+k)} \text {. }
$$

\section{Present Results}

\subsection{The Reference Quantities $\boldsymbol{F}_{k}$}

The essential FIM feature is undoubtedly its being an estimation measure known to obey the Cramer Rao (CR) bound of Equation (2) [1]. Accordingly, since our partial differential equation has multiple solutions, it is natural to follow Jaynes's MaxEnt ideas and select amongst them the one that optimizes the CR bound, that constitutes the informational operative constraint in Fisher's instance. Of course, Jaynes needs to maximize the entropy instead. We will also, without loss of generality, renormalize the reference quantities $F_{k}$. This procedure is convenient because it allows us to regard these quantities as statistical weights that optimize the CR-bound. In other words, our procedure entails that we extremize

$$
\begin{aligned}
f\left(F_{1}, \cdots, F_{M}\right) & =I\left(\left\langle x^{2}\right\rangle-\langle x\rangle^{2}\right) \\
& =\sum_{k=1}^{M} \frac{k}{2}\left[\frac{F_{k}}{\mid\left\langle x^{k}\right\rangle}\right]^{2 / k}\left(\left\langle x^{2}\right\rangle-\langle x\rangle^{2}\right) .
\end{aligned}
$$

with the constraint

$$
\phi\left(F_{1}, \cdots, F_{M}\right)=\sum_{k=1}^{M} F_{k}^{2 / k}=1 .
$$

We are going to apply now the preceding considerations so as to obtain the eigenvalues of the quartic anharmonic oscillator.

\subsection{Quartic Anharmonic Oscillator}

The Schrödinger equation for a particle of unit mass in a quartic anharmonic potential reads,

$$
\left[-\frac{1}{2} \frac{\partial^{2}}{\partial x^{2}}+\frac{1}{2} k x^{2}+\frac{1}{2} \lambda x^{4}\right] \psi=E \psi .
$$

where $\lambda$ is the anharmonicity constant. According to [7], we can ascribe to (28) a Fisher measure and make then the following identifications: $\alpha=8 E, \lambda_{2}=-4 k$, $\lambda_{4}=-4 \lambda$. Accordingly, we have, in the $\left\{\alpha, \lambda_{k}\right\}-$ scenario [Cf. (24)],

$$
\alpha=2 F_{2}^{1 / 2}\left|\lambda_{2}\right|^{1 / 2}+3 F_{4}^{1 / 3}\left|\lambda_{4}\right|^{1 / 3} .
$$

The functions $f$ and $\phi$ defined by (26) and (27), respectively, can here be recast [using (25)] as 


$$
\begin{gathered}
f\left(F_{2}, F_{4}\right)=F_{2}+2 F_{2}^{1 / 2} F_{4}^{1 / 3}\left|\lambda_{2}\right|^{-1 / 2}\left|\lambda_{4}\right|^{1 / 3}, \\
\phi\left(F_{2}, F_{4}\right)=F_{2}+F_{4}^{1 / 2} .
\end{gathered}
$$

After these preparatory moves we can recast our methodology in a convenient specialized fashion, suitable for the task at hand. We just face the simple two-equations system:

$$
\left\{\begin{array}{l}
\nabla f\left(F_{2}, F_{4}\right)=\mu \nabla \phi\left(F_{2}, F_{4}\right) \\
\phi\left(F_{2}, F_{4}\right)=1
\end{array}\right.
$$

where $\nabla \equiv\left(\partial_{F_{2}}, \partial_{F_{4}}\right)$. Straightforward solution of it yields

$$
\begin{aligned}
& F_{2}^{-1 / 2}\left(1-F_{2}\right)^{-1 / 3}\left(7 F_{2}-3\right)=3\left|\lambda_{2}\right|^{1 / 2}\left|\lambda_{4}\right|^{-1 / 3}, \\
& F_{4}=\left(1-F_{2}\right)^{2},
\end{aligned}
$$

from which we obtain $F_{2}$ and $F_{4}$. Substituting them into (29) we determine $\alpha$ and, of course, the eigenvalue $E=\alpha / 8$. Consider now our SE (28), taking $k=1$ and a given value of $\lambda(0.0001 \leq \lambda \leq 10000)$. The function [Cf. Equation (26)] $f\left(F_{2}, F_{4}\right)=I\left\langle x^{2}\right\rangle$ exhibits, as a function of its arguments, a unique "critical" point that satisfies (31). Using $f=f_{\text {critical }}$, that optimizes the CRbound, we find a ground-state eigenvalue that is in good agreement the literature. See some interesting results in Table 1.

In this way, after properly dealing with (24), with the $F_{k}$ regarded as "FIM statistical weights" that optimize the Cramer Rao inequalities, we determine $\alpha$ as a function of the $\lambda_{k}$ without passing first through a Schrödinger equation, which is a notable aspect of the present approach. Interestingly enough, the Cramer-Rao inequality us equivalent to the quantum uncertainty principle (see the Appendix for details and references). Thus, our methodology actually employs Heisenberg's celebrated principle to pick up just one solution among the several ones that our partial differential equation possesses.

\section{Conclusions}

On the basis of a variational principle based on Fisher's information measure, free of adjustable parameters, we have obtained the Schrödinger energy-eigenvalues for the fundamental state of the quartic anharmonic oscillator (for several anharmoniticy-values). Our theoretical results, obtained without passing first through a Schrödinger equation, are in a good agreement with those of the literature. This constitutes an illustration of the power of information-related tools in analyzing physical problems.

Thus, we have in this communication introduced a new general technique for eigenvalue-problems of linear operators, whose use seems to constitute a promising
Table 1. Ground-state eigenvalues of the SE (28) for $k=1$ and several values of the anharmonicity constant $\lambda$. The values of the second column correspond to those one finds in the literature, obtained via a numerical approach to the SE. These results, in turn, are nicely reproduced by some interesting theoretical approaches that, however, need to introduce and adjust some empirical constants [9]. Our values, in the third column, are obtained by means the present theoretical, parameter-free procedure. The fourth column displays the associated Cramer-Rao bound.

\begin{tabular}{cccc}
\hline$\lambda$ & $E_{\text {num }}$ & $E=\alpha / 8$ & $f=I\left\langle x^{2}\right\rangle$ \\
\hline 0.0001 & 1.000074 & 1.000074 & 1.000059 \\
0.001 & 1.000748 & 1.000739 & 1.000591 \\
0.01 & 1.007373 & 1.007263 & 1.005824 \\
0.1 & 1.065285 & 1.063047 & 1.051255 \\
1 & 1.392351 & 1.353533 & 1.296590 \\
10 & 2.449174 & 2.213973 & 2.040974 \\
100 & 4.999417 & 4.212932 & 3.782394 \\
1000 & 10.639788 & 8.587748 & 7.599439 \\
\hline
\end{tabular}

venue, given the results here displayed.

\section{Acknowledgements}

This work was partially supported by the Projects FQM2445 and FQM-207 of the Junta de Andalucia (Spain, EU). Acknowledges support from the Senior Grant CEI Bio-Tic GENIL-SPR.

\section{References}

[1] B. R. Frieden, "Science from Fisher Information: A Unification,” Cambridge, University Press, Cambridge, 2004. doi:10.1017/CBO9780511616907

[2] B. R. Frieden, A. Plastino, A. R. Plastino and B. H. Soffer, "Fisher-Based Thermodynamics: Its Legendre Transform and Concavity Properties,” Physical Review E, Vol. 60, 1999, pp. 48-55. doi:10.1103/PhysRevE.60.48

[3] M. Reginatto, "Derivation of the Equations of Nonrelativistic Quantum Mechanics Using the Principle of Minimum Fisher Information,” Physical Review E, Vol. 58, 1998, pp. $1775-1778$.

[4] S. P. Flego, B. R. Frieden, A. Plastino, A. R. Plastino and B. H. Soffer, "Non-Equilibrium Thermodynamics and Fisher Information: Sound Waves Propagation in a Dilute Gas,” Physical Review E, Vol. 68, No. 16, 2003, pp. 105115.

[5] S. P. Flego, A. Plastino and A. R. Plastino, "Legendretransform Structure Derived from Quantum Theorems," Physica A, Vol. 390, 2011, pp. 2276-2281. doi:10.1016/j.physa.2011.02.019

[6] S. P. Flego, A. Plastino and A. R. Plastino, "Special Fea- 
tures of the Relation between Fisher Information and Schroedinger Eigenvalue Equation,” Journal of Mathematical Physics, Vol. 52, No. 8, 2011, pp. 2103-2116. doi:10.1063/1.3625265

[7] S. P. Flego, A. Plastino and A. R. Plastino, "Inferring an Optimal Fisher Measure,” Physica A, Vol. 390, 2011, pp. 4702-4712. doi:10.1016/j.physa.2011.06.050

[8] F. T. Hioe and E. W. Montroll, "Quantum Theory of Anharmonic Oscillators I. Energy Levels of Oscillators with Positive Quartic Anharmonicity,” Journal of Mathematical Physics, Vol. 16, 1975, pp. 1945-1950. doi:10.1063/1.522747

[9] C. M. Bender and T. T. Wu, "Anharmonic Oscillator," Physical Review, Vol. 184, 1969, pp. 1231-1260.
doi:10.1103/PhysRev.184.1231

[10] K. Banerjee. "W. K. B. Approximation and Scaling," Proceedings of the Royal Society A, Vol. 363, 1978, pp. 147-151.

[11] R. P. Feynman, "Forces in Molecules," Physical Review, Vol. 56, 1939, pp. 340-343.

[12] A. Desloge, “Thermal Physics,” Holt, Rinehart and Winston, New York, 1968.

[13] W. Greiner and B. Müller, "Quantum Mechanics. An Introduction,” Springer, Berlin, 1988.

[14] P. M. Mathews and K. Venkatesan, "A Textbook of Quantum Mechanics,” Tata McGraw-Hill Publishing Company Limited, New Delhi, 1986. 


\section{Appendix: Cramer-Rao and Uncertainty Principle}

It is well known that the Cramer-Rao inequality may be regarded as an expression of Heisenberg's Uncertainty Principle (See, for instance, [1]). Remember that a precise statement of the position-momentum uncertainty principe reads [14]

$$
(\Delta x)(\Delta p) \geq \frac{\hbar}{2} \text { or }(\Delta x)^{2}(\Delta p)^{2} \geq \frac{\hbar^{2}}{4},
$$

where

$$
\begin{aligned}
& (\Delta x)^{2}=\left\langle(x-\langle x\rangle)^{2}\right\rangle=\left\langle x^{2}\right\rangle-\langle x\rangle^{2} \\
& (\Delta p)^{2}=\left\langle(p-\langle p\rangle)^{2}\right\rangle=\left\langle p^{2}\right\rangle-\langle p\rangle^{2} .
\end{aligned}
$$

In a one-dimensional configuration-space, if $\psi$ is a normalizable real wave function,

$$
\begin{aligned}
\langle p\rangle & =\left\langle-i \hbar \frac{\partial}{\partial x}\right\rangle=-i \hbar \int \psi \frac{\partial}{\partial x} \psi \mathrm{d} x \\
& =-i \frac{\hbar}{2} \int \frac{\partial}{\partial x} \psi^{2} \mathrm{~d} x=0,
\end{aligned}
$$

$$
\left\langle p^{2}\right\rangle=\left\langle-\hbar^{2} \frac{\partial^{2}}{\partial x^{2}}\right\rangle=-\hbar^{2} \int \psi \frac{\partial^{2}}{\partial x^{2}} \psi \mathrm{d} x .
$$

Substituting (35) and (36) in (34) and using (7) leads to the above mentioned connection between the uncertainty in momentum $\Delta p$ and the Fisher's measure $I$, i.e.,

$$
(\Delta p)^{2}=-\hbar^{2} \int \psi \frac{\partial^{2}}{\partial x^{2}} \psi \mathrm{d} x=\frac{\hbar^{2}}{4} I .
$$

If this relation is substituted into (32) we immediately arrive to the the CR-bound,

$$
(\Delta x)^{2}(\Delta p)^{2} \geq \frac{\hbar^{2}}{4} \rightarrow I(\Delta x)^{2} \geq 1 .
$$

Coming now back to the $\left\{\alpha, \lambda_{k}\right\}$-scenario, one easily ascertains that Equation (26) can be given a clear "Heisenberg's aspect"

$$
\begin{aligned}
f\left(F_{1}, \cdots, F_{M}\right)= & \sum_{k=1}^{M} \frac{k}{2}\left[F_{k}\left|\lambda_{k}\right|\right]^{2 /(2+k)} \\
& \left(F_{2}^{1 / 2}\left|\lambda_{2}\right|^{-1 / 2}-F_{1}^{4 / 3}\left|\lambda_{1}\right|^{-2 / 3}\right) .
\end{aligned}
$$

